\title{
Structure and function of DEAH-box helicase 32 and its role in cancer (Review)
}

\author{
QINGCHUN WEI ${ }^{1 *}$, JINTING GENG $^{2 *}$, YONGQUAN CHEN $^{2}$, HUAYUE LIN $^{3}$, \\ JIAJIA WANG ${ }^{3}$, ZANXI FANG $^{3}$, FEN WANG $^{4}$ and ZHONGYING ZHANG ${ }^{1,2}$

\begin{abstract}
${ }^{1}$ State Key Laboratory of Molecular Vaccinology and Molecular Diagnostics, School of Public Health, Xiamen University, Xiamen, Fujian 361102; ${ }^{2}$ Xiamen Key Laboratory of Biomarker Translational Medicine,

${ }^{3}$ Center of Clinical Laboratory, Xiamen University Affiliated Zhongshan Hospital, Xiamen, Fujian 361104, P.R. China; ${ }^{4}$ Center for Cancer and Stem Cell Biology, Institute of Biosciences and Technology,
\end{abstract} \\ Center of Medical Laboratory of Xiamen Humanity Hospital, Fujian Medical University, Xiamen, Fujian 361009; \\ Texas A\&M Health Science Center, Houston, TX 77030, USA
}

Received August 16,2020; Accepted December 23, 2020

DOI: $10.3892 / \mathrm{ol} .2021 .12643$

\begin{abstract}
DEAH-box helicase 32 (DHX32) is an RNA helicase with unique structural characteristics that is involved in numerous biological processes associated with RNA, including ribosome biosynthesis, transcription, mRNA splicing and translation. Increasing evidence suggests that abnormal DHX32 expression contributes to cancer initiation and development, due to dysregulated cell proliferation, differentiation, apoptosis and other processes. In the current review, the discovery, structure and function of DHX32, as well as the association between abnormal DHX32 expression and tumors are discussed. DHX32 expression is downregulated in acute lymphoblastic leukemia, but upregulated in solid tumors, including colorectal and breast cancer. Furthermore, DHX32 expression levels are associated with the pathological and clinical features of the cancer. Therefore, DHX32 may serve as a novel liquid biopsy marker for auxiliary diagnosis and prognosis screening, as well as a possible target for cancer therapy. The molecular mechanism underlying the contribution of DHX32 towards the initiation and development of cancer requires further investigation for the development of anticancer treatments based on manipulating DHX32 expression and function.
\end{abstract}

Correspondence to: Professor Zhongying Zhang, State Key Laboratory of Molecular Vaccinology and Molecular Diagnostics, School of Public Health, Xiamen University, 4221-117 Xiang'an South Road, Xiang'an, Xiamen, Fujian 361102, P.R. China

E-mail: zhangzy1121@xmu.edu.cn

${ }^{*}$ Contributed equally

Key words: DHX32, RNA helicases, biomarkers, tumor diagnosis and prognosis, tumor treatment

\section{Contents}

1. Introduction

2. Discovery of RNA helicase DHX32

3. Structure of DHX32 gene

4. Helicase activity of DHX32

5. Subcellular location of DHX32

6. DHX32 and cell differentiation

7. DHX32 and cancer

8. Perspective and future direction

\section{Introduction}

RNA helicases are a class of enzymes that regulate the structure and function of RNAs by utilizing the energy of nucleoside triphosphate (NTP) to unwind the secondary structure of RNAs, which interferes with protein interactions in certain cases (1). The amino acid sequence of RNA helicases is characterized by the existence of two typical RecA-like helicase domains, the RecA1 domain and the RecA2 domain (Fig. 1). The conserved motifs I, Ia, Ib and III are located in the RecA1 domain, whereas the conserved motifs IV, V and $\mathrm{VI}$ are located in the RecA2 domain (2). The non-conserved $\mathrm{N}$-terminal domain (NTR) and C-terminal domain (CTR) are located at both ends of the catalytic core domain. The NTR and CTR domains determine the expression pattern, subcellular localization and substrate specificity of the enzyme $(3,4)$. According to the amino acid sequence and structure, RNA helicases can be divided into six super-families (SF) 1-6, among which SF2 contains the most members (5). SF2 contains 8-9 highly conserved motifs, including motifs Q, I, Ia, Ib, II, III, IV, V and VI, each of which display a distinct function. Motifs I, II, VI and Q bind to NTP and catalyze hydrolysis; motifs Ia, Ib and IV primarily bind to RNA substrates; and motifs III and V connect the NTP binding and RNA recognition sites, promoting NTP-dependent RNA unwinding $(6,7)$. Based on the amino acid sequence of conserved motif II, 
SF2 are divided into two subfamilies: DEAD-box helicase and DEAH-box helicase (DEAH), which are named after the amino acid sequence Asp(D)-Glu(E)-Ala(A)-Asp(D)/His(H) of motif II, referred to as DDX and DHX, respectively (8). Non-conserved domains and conserved motifs 8-9 determine several important biological properties of RNA helicases, including splicing, transportation, translation initiation, RNA degradation and ribosome synthesis (Fig. 1) (9).

Emerging evidence suggests that numerous RNA helicases are dysregulated in tumor tissues: DDX1 is highly expressed in neuroblastoma and retinoblastoma (10) and breast cancer (11); DDX2A is highly expressed in melanoma (12) and hepatocellular carcinoma (13); DDX3 is upregulated in breast (14) and liver cancer (15); DDX48 is upregulated in gastric cancer (16); DDX43 is upregulated in acute myeloid leukemia (17) and lung cancer (18); and DDX5 and DDX6 are upregulated in colorectal cancer $(19,20)$. In contrast, DDX3 is downregulated in certain types of liver and lung cancer (21). Moreover, DHX32 is downregulated in acute lymphocytic leukemia, but upregulated in colorectal and breast cancer (Table I) $(10,12,13,17-36)$. The aforementioned studies indicate that the abnormal expression of RNA helicases is significantly associated with cancer initiation and development.

\section{Discovery of RNA helicase DHX32}

DHX32 is a helicase of the DHX family that was discovered in 2002 by Abdelhaleem (37) based on the similarity to the amino acid sequence of the DDX15 helicase domain. The potentially encoded sequence FLJ10889 (accession no. XM 045832) was identified by performing a National Center for Biotechnology Information non-redundant protein database search, which was then used to search the expressed sequence tag database to obtain the overlapping expressed sequence tags (AL599197) with 5' ends. The full-length sequence was confirmed via reverse transcription-PCR amplification and sequencing, and DHX32 was identified as the DEAH helicase homolog. At the same time, a variant transcript (DHX32 $\triangle 5)$ with a 243-bp deletion in exon 5 was also identified. Both DHX32 and DHX32 $\Delta 5$ were confirmed by cloning from HL-60 bone marrow leukemia cells $(37,38)$.

\section{Structure of DHX32 gene}

Human DHX32 coding sequence (Gene ID: 55760) is located on chromosome 10q26.2. The gene contains 12 exons and the full-length mRNA (Genbank: AF427340) is $3071 \mathrm{bp}$ in length (Fig. 2). Exon 1 contains a $\mathrm{CpG}$ island, which serves as a potential distant promoter. Exon 2 contains the proximal promoter, which contains the TATA box sequence. DHX32 RNA in the thymus and viscera are transcribed from both the distal (Genbank: XM 017016404.1) and proximal promoters (Genbank: NM 018180.2). DHX32 transcripts in the bone marrow are only transcribed from the proximal promoter (Genbank: NM 018180.2) (38). Although the two set of DHX32 RNA contain different 5'-untranslated regions, they share the same translational initiation ATG codon. The use of different promoters represents a layer of regulation for the tissue- and developmental stage-specific gene expression of DHX32 (39).
The coding sequence of DHX32 encompasses 11 exons. DHX32 protein contains 743 amino acids, with a molecular weight of $84 \mathrm{kDa}$ (Genbank: NM_018180.2). In addition, alternative splicing of DHX32 mRNA yields the DHX32 $\triangle 5$ variant, which has a 243-bp deletion in exon 5 (Genbank: AF427341). The corresponding protein has a deletion of 81 residues (284aa-364aa) and a molecular weight of $75 \mathrm{kDa}$. However, which promoter is used for this variant mRNA has not yet been identified (Fig. 2) (38).

\section{Helicase activity of DHX32}

Compared with other members of the DEAH family, DHX32 has a unique helicase domain sequence that is characterized by the presence of amino acid substitutions in all eight motifs (Fig. 3) (37). Motifs I and II are the ATP-binding sites. Structural-based analyses demonstrate that not only the lysine in motif I, but also the aspartate, glutamine and histidine residues in motif II are critical for enzymatic activity (40). Except for the glutamine residue in motif II, which is replaced by aspartate, all other key amino acids are conserved. Therefore, it is likely that DHX32 is ATPase active. However, compared with the traditional DEAH family, the serine-alanine-threonine (SAT) domain in motif III of the DHX32 is replaced by the serineserine-proline (SSP) domain (Fig. 3), which is involved in ATP hydrolysis and RNA unwinding (41). Several key residues in the QRxGRxGR sequence of motif VI are not conserved in DHX32. Since the QRxGRxGR domain is the key site for nucleic acid substrate binding (42), it is likely that DHX32 may not display substrate binding and thus, will not display helicase activity. In addition to containing a SSP motif III, DHX32 $\Delta 5$ also has a deletion of motif V (Fig. 3), which connects the ATP binding and RNA recognition sites required for the unwinding of RNA (7). Therefore, it is speculated that DHX $32 \Delta 5$ displays a lower helicase activity compared with DHX32.

\section{Subcellular location of DHX32}

DHX32 is widely distributed in human tissues, including the bone marrow, thymus, spleen, rectum and breast. Confocal microscopy revealed that DHX32 is localized in the nucleus and mitochondria of HeLa cells (43). RNA helicases that regulate mRNA transcription and splicing are located in the nucleus, whereas those that regulate translation are located in the cytoplasm (44). The location of DHX32 in the nucleus and mitochondria reflects the diversity of its functions. Electron microscope immunocytochemistry revealed that DHX32 is localized in the mitochondria of mouse hepatocytes (45), and is localized in the nucleus and the inner mitochondrial membrane in HL-60 leukemia cells (43). Iborra et al (46) demonstrated that newly synthesized RNA molecules of mitochondria accumulate along the inner membrane. Alli et al (43) double stained cells with anti-bromouridine and anti-DHX32 antibodies, demonstrating DHX32 mitochondria localization, which was similar to the position of newly synthesized RNA in mitochondria. Collectively, the aforementioned studies indicate that DHX32 is involved in regulating mitochondrial gene expression. Heat shock protein 60 (Hsp60), an important molecular chaperone, is primarily distributed in the mitochondria in Jurkat cells. Chen et al (47) reported that when 

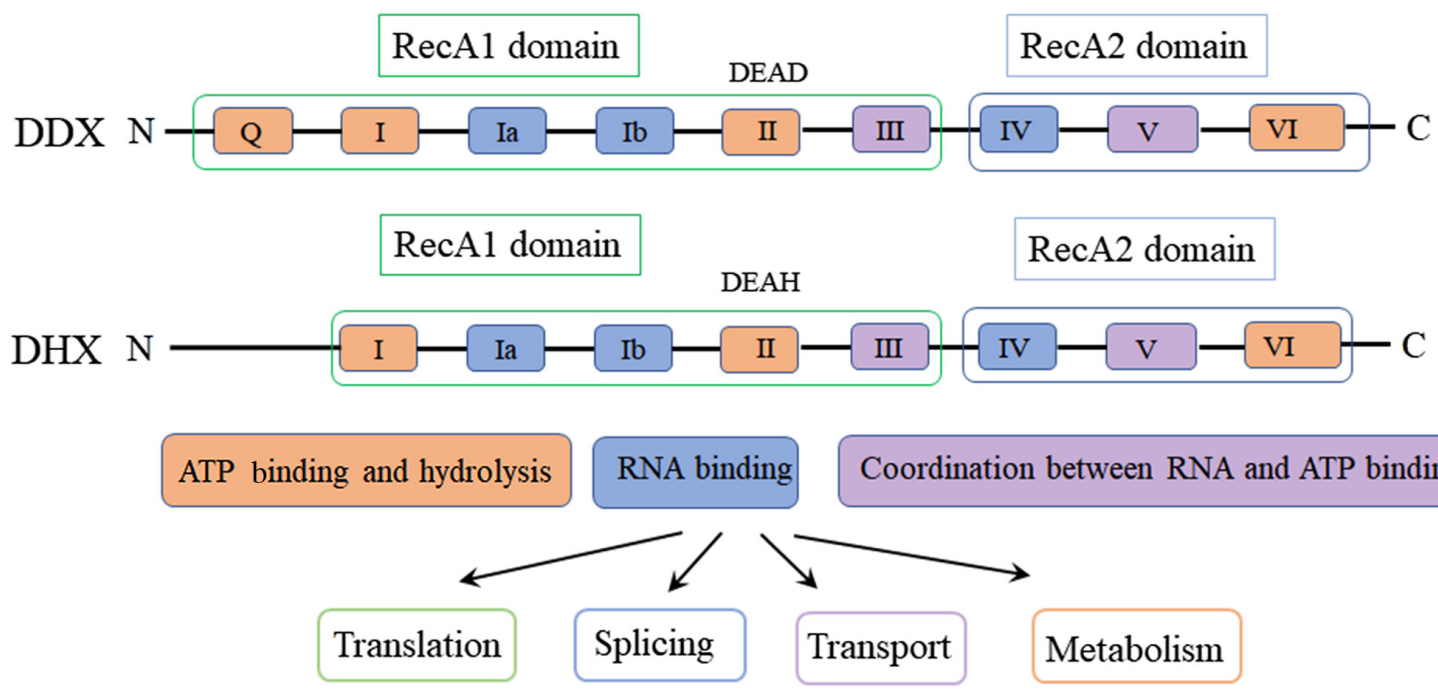

Figure 1. Conserved functional domains in DEAD/H helicases. The motifs are colored according to their biochemical functions: Orange, a motif that binds and hydrolyzes ATP; purple, a motif that regulates the binding of RNA to ATP; blue, a motif that binds double-stranded RNA of a nucleic acid substrate.

Table I. Association between RNA helicases and tumors.

\begin{tabular}{lll}
\hline RNA helicases & \multicolumn{1}{c}{ Associated tumor } & Expression level \\
\hline DDX1 & Neuroblastoma, retinoblastoma & Upregulated \\
DDX2A & Melanoma, hepatocellular carcinoma & Upregulated \\
DDX4 & Ovarian cancer, blood-derived cancer & Upregulated \\
DDX3 & Breast, liver cancer/liver, lung cancer & Up/downregulated \\
DDX5 & Colorectal, prostate, breast cancer & Upregulated \\
DDX6 & Gastric cancer, colorectal cancer & Upregulated \\
DDX11 & Invasive melanoma, lung adenocarcinoma & Upregulated \\
DDX17 & Colorectal cancer & Upregulated \\
DDX39 & Hepatocellular carcinoma, lung cancer & Upregulated \\
DDX43 & Acute myeloid leukemia, lung cancer & Upregulated \\
DDX48 & Gastric cancer, vaginal carcinoma & Upregulated \\
DDX53 & Gastric, cervical and lung cancer & Upregulated \\
DHX9 & Lung cancer & Upregulated \\
DHX15 & Glioma & Upregulated \\
DHX32 & Colorectal cancer/acute lymphocytic leukemia & Up/downregulated \\
\hline
\end{tabular}

DHX32 is highly expressed, Hsp60 was transferred from the mitochondria to the cytoplasm in Jurkat cells. Therefore, DHX32 may also regulate the redistribution of mitochondrialassociated proteins, and then, may regulate gene expression in both the nucleus and mitochondria.

\section{DHX32 and cell differentiation}

DHX32 expression is different in normal lymphoid tissues. In lymphoid follicles, DHX32 expression is higher in lymphocytes at the germinal center compared with the mantle region. In the spleen, lymphocytes of red pulp express higher levels of DHX32 compared with the white pulp. In the thymus, DHX32 expression is higher in the thymic medulla compared with the thymic cortex (38). The aforementioned results indicate that DHX32 expression in normal lymphoid tissues is asso- ciated with lymphocyte activation and differentiation status. Further studies demonstrate that DHX32 expression is low in precursor $\mathrm{T}$ lymphoblastic lymphoma derived from precursor cells of acute lymphoblastic leukemia, and high in large B-cell lymphoma derived from mature lymphocytes. Furthermore, DHX32 expression in CD4 and CD8 double-negative cells and double-positive cells is significantly lower compared with single-positive cells, indicating that DHX32 expression is positively correlated with lymphocyte proliferation and differentiation (48). Overall, DHX32 expression is closely associated with cell proliferation, differentiation and apoptosis.

\section{DHX32 and cancer}

DHX32 and hematological tumors. In 2002, by using RNA hybridization, Abdelhaeem (37) demonstrated that DHX32 


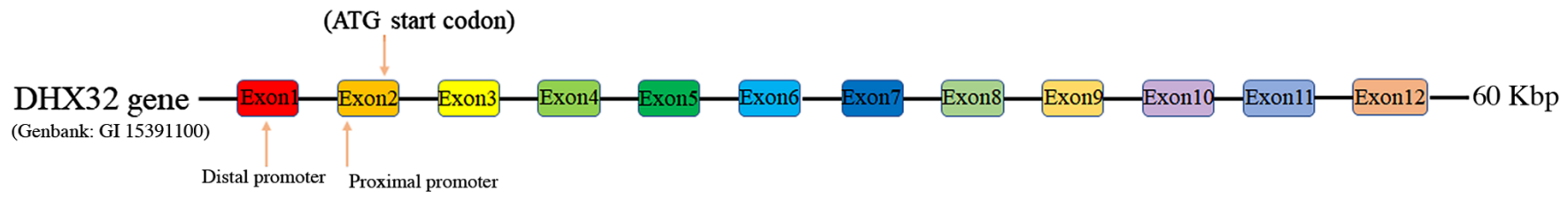

\section{DHX32 mRNA transcripts}

Exon 1 transcript

(Genbank: BC068471)

Exon 2 transcript

(Genbank: NM_018180.2)

Deleted Exon 5 transcript

(GenBank: AF427341)

Deleted Exon 5 transcript (GenBank: AF427341)

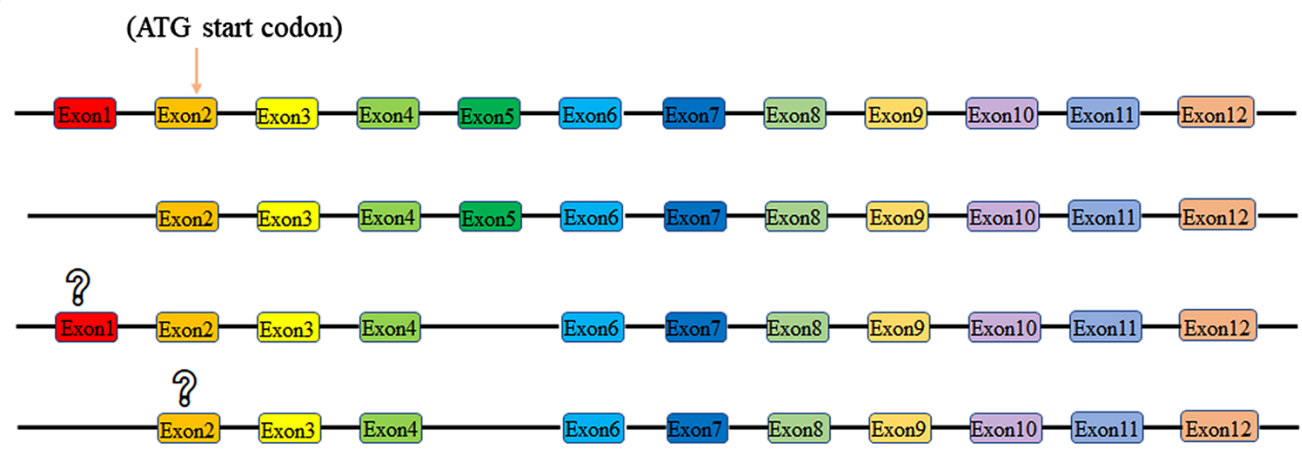

Figure 2. DHX32 gene structures and its transcripts. The DHX32 gene contains 12 exons. It has two promoters: Distal promoter and the proximal promoter. The mRNA transcripts transcript from the two promoters differ at the 5 ' end. The translational initiating ATG codon is located at exon 2 . Thus, the two mRNA encode for the same protein. The alternative splicing leads to inclusion or exclusion of exon 5 . However, the 5'-end noncoding sequence has not been identified in the variants that lack exon 5 .

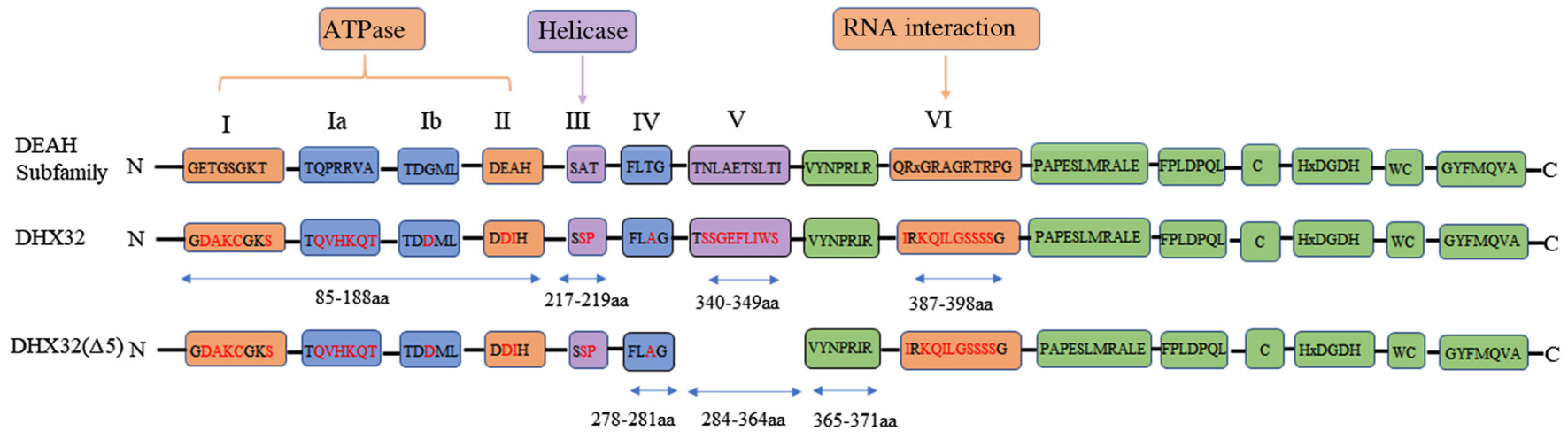

Figure 3. Schematic diagram of the homology regions of DEAH subfamily, DHX32 and DHX32 45 . The unique amino acids in DHX32 are marked in red. The alternative spliced motif V (340-349) is absent in the DHX32 $\triangle 5$ splice variant.

was downregulated in acute lymphocytic leukemia cells and patients' cancer cells. T cell lines, precursor B cell lines and precursor B cell lymphocytic leukemia samples from patients also displayed downregulation of DHX32 expression, indicating that DHX32 downregulation is not restricted to a specific lymphocyte lineage. Interestingly, there were no significant differences in other DEAH expression levels, including DDX15 and DDX9, in the myeloid and lymphoid lines (37), suggesting that downregulated RNA helicase expression is DHX32specific. In 2005, immunohistochemical staining demonstrated that DHX32 expression was low in follicular lymphoma and Burkitt lymphoma, but is high in mantle cell lymphoma, large B-cell lymphoma and Reed-Steenberg cells of nodular sclerotic Hodgkin lymphoma with a higher degree of malignancy (38). The aforementioned finding provided evidence that DHX32 expression is dysregulated in lymphoma, and the expression level is significantly different in various types of tumor tissues. The higher the malignancy, the more significant the alterations in DHX32 expression levels are, which suggests the potential of
DHX32 to serve as a novel biomarker for lymphoma prognosis. A study recruiting 28 patients with primary chemotherapyresistance leukemia was conducted by McNeer et al (49). The aforementioned study reported that DHX32 gene deletions were the cause of chemotherapy-resistance in pediatrics with acute myeloid leukemia. Moreover, the frequency of DHX32 mutant allele was increased from 14 to $39 \%$ after chemotherapy. The cBioPortal of TCGA pan-cancer repository (RRID: SCR_014555, URL: https://www.cbioportal.org/) was used to explore DHX32 functionality in big data. There were 31 studies (12,845 samples) in myeloid leukemia and lymphoid leukemia. These data were analyzed by using a log-rank test (R package survival) and found that the DHX32 missense mutations are associated with the poor prognosis of patients in myeloid leukemia and lymphocytic leukemia. These findings confirm that DHX32 is abnormally expressed in hematological tumors and is associated with the malignancy of the tumor, further demonstrating the potential of DHX32 as a prognostic biomarker in leukemia. 


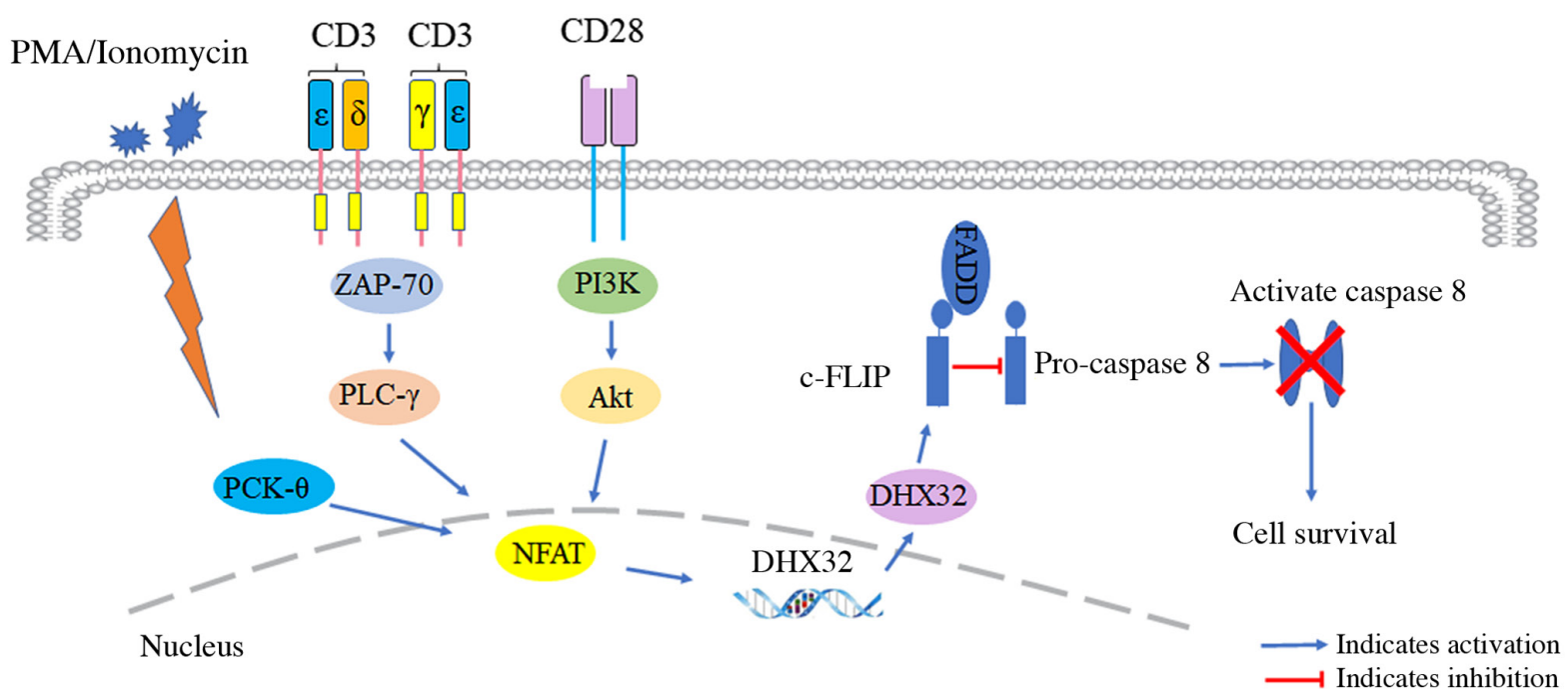

Figure 4. Regulation and function of DHX32 gen.

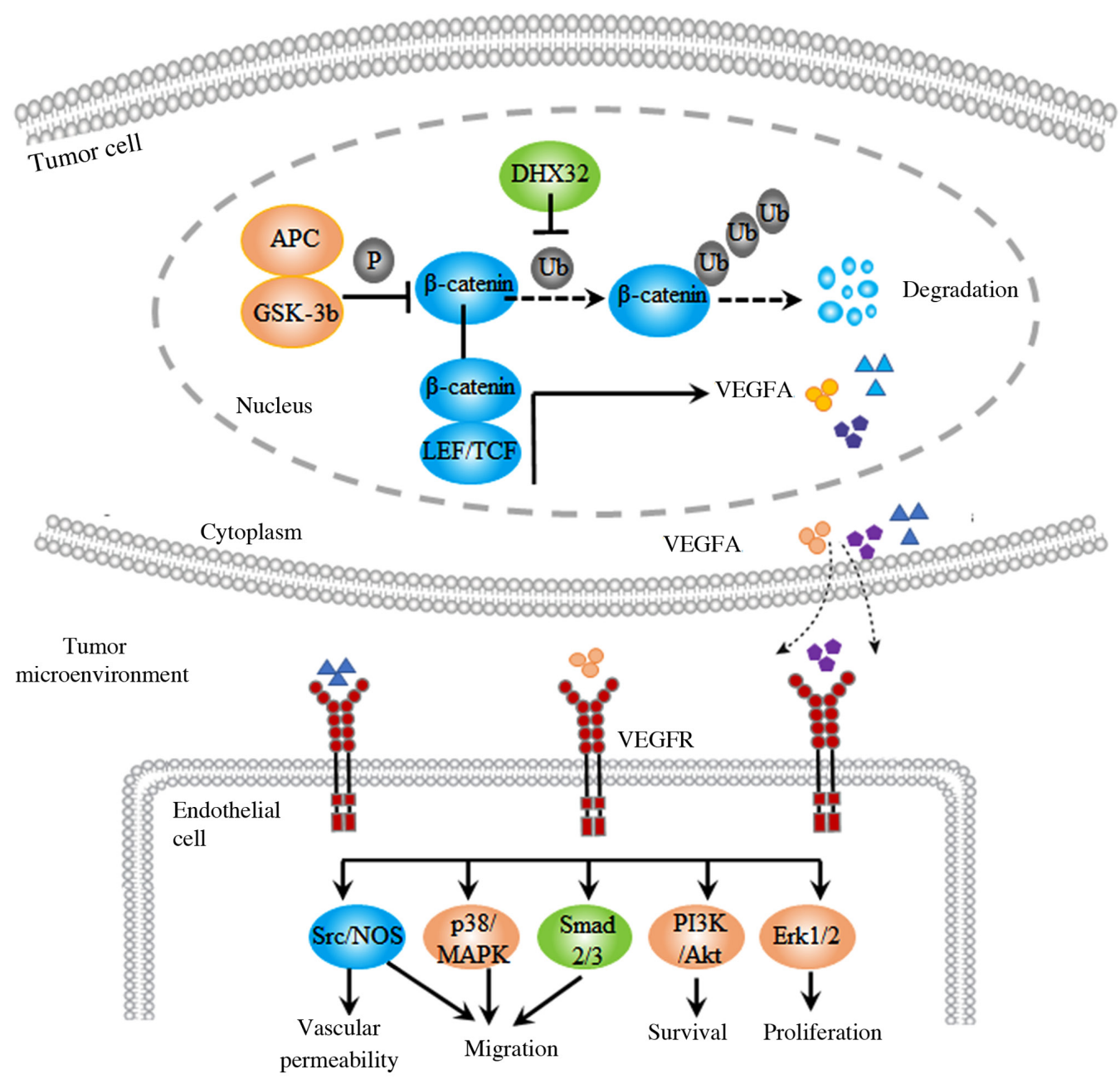

Figure 5. Prediction mechanism of DHX32 regulates tumor angiogenesis.

In 2006, it was reported that DHX32 expression was significantly upregulated when co-stimulated by CD3 and CD28 antibodies, or stimulated by phorbol 12-myristate 13-acetate, or ionomycin alone in Jurkat cells with low background DHX32 expression (50). Noteworthy, only the mRNA isoform transcribed from the proximal promoter was expressed in activated Jurkat cells. The proximal promoter of DHX32 has a binding site for the nuclear transcription factor nuclear factor of activated T-cells (NFAT) (50). It was speculated that DHX32 expression could be regulated by NFAT during T cell activation. 
Forced expression of DHX32 in Jurkat cells did not affect cell proliferation and the response to chemotherapeutic agents, including actinomycin D and etoposide. However, forced DHX32 expression downregulated the expression of antiapoptotic protein c-FLIP was associated with the Fas signaling pathway and promoted apoptosis. In normal peripheral blood lymphocytes, co-stimulation with phytohemagglutinin, and CD3 and CD28 antibodies upregulated the expression of DHX32 and c-FLIP, and inhibited apoptosis (51). Therefore, it has been proposed that DHX32 overexpression can increase the response to apoptosis associated with Fas signaling by regulating the expression of c-FLP protein, contributing to tumorigenesis (Fig. 4).

DHX32 and solid tumors. In 2009, by using mRNA differential display PCR to screen the progression and metastasis-specific markers of colorectal cancer, Huang et al (52) reported that DHX32 expression in colorectal cancer was higher compared with adjacent non-cancerous tissues, and the expression was significantly associated with tumor thrombus formation, lymph node metastasis, histological grade and Dukes stage of the cancer, indicating that abnormal DHX32 expression may be involved in colorectal cancer initiation and progression. Furthermore, Lin et al (53) discovered that DHX32 promoted colorectal cell proliferation, invasion and migration. The quantitative PCR analyses identified that genes involved in cell proliferation, apoptosis, invasion and migration were regulated by DHX32, which included VEGFA. Further research demonstrated that DHX32 increased the transcriptional regulatory activity of $\beta$-catenin, which is an upstream regulator of the VEGFA gene. DHX32 upregulation inhibited $\beta$-catenin ubiquitination, prolonged the half-life of $\beta$-catenin and improved the stability of $\beta$-catenin, thus promoting VEGFA expression. DHX32 knockdown significantly inhibited colorectal cancer xenograft growth and angiogenesis in nude mice (54). The results indicated that DHX32 induces VEGFA expression by augmenting $\beta$-catenin signaling, thereby promoting angiogenesis in colorectal cancer (Fig. 5).

In solid tumors, DHX32 expression has been evaluated in pathological samples. In colorectal cancer, the association between DHX32 expression levels and clinical pathology was analyzed in 139 colorectal cancer tissues and 93 corresponding adjacent non-cancerous tissues via immunohistochemistry. The results demonstrated that DHX32 was upregulated in colorectal cancer tissues compared with adjacent tissues, and DHX32 expression was associated with tumor microvascular density, degree of differentiation and poor patient prognosis (54). Similarly, in a cohort of 193 patients with breast cancer, DHX32 mRNA and protein expression levels were increased compared with adjacent non-cancerous tissues, and there was statistical significance between DHX32 expression and breast cancer clinical stage, histological grade, lymph node metastasis and proliferation marker Ki-67. Kaplan-Meier survival analysis revealed that increased DHX32 expression was associated with poor prognosis in breast cancer. Moreover, the Cox proportional hazard model suggested that DHX32 expression was an independent prognostic factor for low survival and disease-free survival in breast cancer (55). In summary, DHX32 expression is upregulated in colorectal and breast cancer, and its expression is significantly associated with the occurrence, development and poor prognosis of the cancer, indicating that DHX32 might serve as a novel biomarker for colorectal and breast cancer.

\section{Perspective and future direction}

DHX32 has a unique helicase domain that contains eight conserved motifs that are different from other RNA helicases. The variation of these conserved amino acid residues does not affect the ATPase activity, but does alter the unwinding activity. The function of RNA helicase does not simultaneously depend on ATPase activity and unwinding activity. Alternative splicing is a post-transcriptional modification process that allows a gene to encode multiple proteins that have similar structures but different functions. Abnormal splicing is closely associated with cancer initiation and progression. Some splicing variants are specifically expressed in tumor tissues and can be used as biomarkers for tumor diagnosis and targets for cancer treatments (56). DHX32 has two isomers; however, whether their ATPase and helicase activities are similar, whether they share same expression pattern in cancer and how they affect cancer cell growth, differentiation and survival are not completely understood and require further investigation.

DHX32 expression is significantly upregulated in colorectal and breast cancer. In addition, the abundance of DHX32 in colorectal and breast cancer is associated with the clinical and pathological features of the patients. DHX32 does not have a signal peptide and is an intracellular protein. However, several non-secretory proteins, including DDX48 helicase (16) and hsp70 $(57,58)$, can be detected in the circulation and serve as biomarkers for cancer, since overexpressed proteins in cancer cells are often discharged into the circulation. Thus, whether DHX32 proteins can be detected in peripheral blood of the patients requires further investigation. In addition, additional bioinformatics analyses are required to establish the association between the abundance of DHX32, and clinical and pathological features of samples. Therefore, developing a novel method for assessing DHX32 protein in peripheral blood and liquid biopsy samples, and determining whether DHX32 can serve as a biomarker for screening, diagnosis and prognosis of colorectal and breast cancer are important for translational medicine.

DHX32 expression is dysregulated in cancer cells, indicating its potential as a target for anticancer treatment. However, this is an under-explored area of research as the majority of current efforts to develop drugs targeting helicase are focused on DDX3 $(59,60)$. RK-33 is a small molecule inhibitor that binds to the ATP-binding site of DDX3, inhibiting its enzyme activity. Preclinical studies have reported that RK-33 is a promising drug for treating breast cancer. It was hypothesized that DHX32 may serve as a target for controlling the Wnt signaling pathway, thus could be used for the treatment of colorectal and breast cancer. However, the structure of DHX32 has not been previously reported. Therefore, the molecular structure of DHX32 should be identified to facilitate the development of drugs to target DHX32.

DHX32 is a multifunctional protein that regulates ribosome biosynthesis, transcription, splicing and translation 
of mRNA, as well as numerous other biological activities. DHX32 serves important roles in cell proliferation, differentiation and apoptosis, promoting cancer initiation and progression, as well as tumor angiogenesis. Interestingly, in human acute lymphoblastic leukemia, DHX32 expression is downregulated, but in colorectal and breast cancer, DHX32 expression is significantly upregulated. The mechanism underlying abnormal DHX32 expression in tumors is not completely understood; therefore, further investigation is required.

\section{Acknowledgements}

Not applicable.

\section{Funding}

This work was supported by the Youth Foundation Project of Fujian Provincial Health Department (grant no. 2014-2-69); Fujian Provincial Health and Family Planning Commission, Fujian, China (grant no. 2016J01626).

\section{Availability of data and materials}

Not applicable.

\section{Authors' contributions}

QW researched the topic literation, designed the study and wrote the initial manuscript. JG, YC, HL, JW, ZF and FW revised for important intellectual content, $\mathrm{ZZ}$ revised for important intellectual content and supervised. All authors read and approved the final manuscript.

\section{Ethics approval and consent to participate}

Not applicable.

\section{Patient consent for publication}

Not applicable.

\section{Competing interests}

The authors declare that they have no competing interests.

\section{References}

1. Jankowsky E, Gross CH, Shuman S and Pyle AM: Active disruption of an RNA-protein interaction by a DExH/D RNA helicase. Science 291: 121-125, 2001.

2. Fairman-Williams ME, Guenther UP and Jankowsky E: SF1 and SF2 helicases: Family matters. Curr Opin Struct Biol 20 313-324, 2010

3. He Y, Andersen GR and Nielsen KH: Structural basis for the function of DEAH helicases. EMBO Rep 11: 180-186, 2010.

4. Smith WA, Schurter BT, Wong-Staal F and David M: Arginine methylation of RNA helicase a determines its subcellular localization. J Biol Chem 279: 22795-22798, 2004.

5. Singleton MR, Dillingham MS and Wigley DB: Structure and mechanism of helicases and nucleic acid translocases. Annu Rev Biochem 76: 23-50, 2007.

6. Marchat LA, Arzola-Rodríguez SI, Hernandez-de la Cruz O, Lopez-Rosas I and Lopez-Camarillo C: DEAD/DExH-Box RNA Helicases in Selected Human Parasites. Korean J Parasitol 53 583-595, 2015
7. Du Pont KE, Davidson RB, McCullagh M and Geiss BJ: Motif $V$ regulates energy transduction between the flavivirus NS3 ATPase and RNA-binding cleft. J Biol Chem 295: 1551-1564, 2020.

8. Robert F and Pelletier J: Perturbations of RNA helicases in cancer. Wiley Interdiscip Rev RNA 4: 333-349, 2013.

9. Abdelhaleem M: RNA helicases: Regulators of differentiation. Clin Biochem 38: 499-503, 2005.

10. Godbout R, Li L, Liu RZ and Roy K: Role of DEAD box 1 in retinoblastoma and neuroblastoma. Future Oncol 3: 575-587, 2007.

11. Taunk NK, Goyal S, Wu H, Moran MS, Chen S and Haffty BG: DEAD box 1 (DDX1) expression predicts for local control and overall survival in early stage, node-negative breast cancer. Cancer 118: 888-898, 2012.

12. Eberle J, Krasagakis K and Orfanos CE: Translation initiation factor eIF-4A1 mRNA is consistently overexpressed in human melanoma cells in vitro. Int J Cancer 71: 396-401, 1997.

13. Shuda M, Kondoh N, Tanaka K, Ryo A, Wakatsuki T, Hada A, Goseki N, Igari T, Hatsuse K, Aihara T, et al: Enhanced expression of translation factor mRNAs in hepatocellular carcinoma. Anticancer Res 20: 2489-2494, 2000.

14. Heerma van Voss MR, Schrijver WAME, Ter Hoeve ND, Hoefnagel LD, Manson QF, van der Wall E, Raman V and van Diest PJ; Dutch Distant Breast Cancer Metastases Consortium: The prognostic effect of DDX3 upregulation in distant breast cancer metastases. Clin Exp Metastasis 34: 85-92, 2017.

15. Wang Z, Shen GH, Xie JM, Li B and Gao QG: Rottlerin upregulates DDX3 expression in hepatocellular carcinoma. Biochem Biophys Res Commun 495: 1503-1509, 2018.

16. Xia Q, Kong XT, Zhang GA, Hou XJ, Qiang H and Zhong RQ: Proteomics-based identification of DEAD-box protein 48 as a novel autoantigen, a prospective serum marker for pancreatic cancer. Biochem Biophys Res Commun 330: 526-532, 2005.

17. Lin J, Chen Q, Yang J, Qian J, Deng ZQ, Qian W, Chen XX, Ma JC, Xiong DS, Ma YJ, et al: DDX43 promoter is frequently hypomethylated and may predict a favorable outcome in acute myeloid leukemia. Leuk Res 38: 601-607, 2014.

18. Ma N, Xu HE, Luo Z, Zhou J, Zhou Y and Liu M: Expression and significance of DDX43 in lung adenocarcinoma. Pak J Pharm Sci 30 (Suppl 4): 1491-1496, 2017.

19. Dai L, Pan G, Liu X, Huang J, Jiang Z, Zhu X, Gan X, Xu Q and Tan N: High expression of ALDOA and DDX5 are associated with poor prognosis in human colorectal cancer. Cancer Manag Res 10: 1799-1806, 2018.

20. Taniguchi K, Sugito N, Kumazaki M, Shinohara H, Yamada N, Matsuhashi N, Futamura M, Ito Y, Otsuki Y, Yoshida K, et al: Positive feedback of DDX6/c-Myc/PTB1 regulated by miR-124 contributes to maintenance of the Warburg effect in colon cancer cells. Biochim Biophys Acta 1852: 1971-1980, 2015.

21. He Y, Zhang D, Yang Y, Wang X, Zhao X, Zhang P, Zhu H, Xu N and Liang S: A double-edged function of DDX3, as an oncogene or tumor suppressor, in cancer progression (Review). Oncol Rep 39: 883-892, 2018.

22. Kim KH, Kang YJ, Jo JO, Ock MS, Moon SH, Suh DS, Yoon MS, Park ES, Jeong N, Eo WK, et al: DDX4 (DEAD box polypeptide 4) colocalizes with cancer stem cell marker CD133 in ovarian cancers. Biochem Biophys Res Commun 447: 315-322, 2014.

23. Schudrowitz N, Takagi S, Wessel GM and Yajima M: Germline factor DDX4 functions in blood-derived cancer cell phenotypes. Cancer Sci 108: 1612-1619, 2017.

24. Janknecht R: Multi-talented DEAD-box proteins and potential tumor promoters: p68 RNA helicase (DDX5) and its paralog, p72 RNA helicase (DDX17). Am J Transl Res 2: 223-234, 2010.

25. Taniguchi K, Iwatsuki A, Sugito N, Shinohara H, Kuranaga Y, Oshikawa Y, Tajirika T, Futamura M, Yoshida K, Uchiyama K, et al: Oncogene RNA helicase DDX6 promotes the process of c-Myc expression in gastric cancer cells. Mol Carcinog 57: 579-589, 2018.

26. Bhattacharya $C$, Wang $X$ and Becker D: The DEAD/DEAH box helicase, DDX11, is essential for the survival of advanced melanomas. Mol Cancer 11: 82, 2012.

27. Li J, Liu L, Liu X, Xu P, Hu Q and Yu Y: The role of upregulated DDX11 as a potential prognostic and diagnostic biomarker in lung adenocarcinoma. J Cancer 10: 4208-4216, 2019.

28. Vychytilova-Faltejskova P, Svobodova Kovarikova A, Grolich T, Prochazka V, Slaba K, Machackova T, Halamkova J, Svoboda M, Kala Z, Kiss I, et al: MicroRNA biogenesis pathway genes are deregulated in colorectal cancer. Int J Mol Sci 20: 20, 2019 
29. Zhang T, Ma Z, Liu L, Sun J, Tang H, Zhang B, Zou Y and Li H: DDX39 promotes hepatocellular carcinoma growth and metastasis through activating Wnt/ $\beta$-catenin pathway. Cell Death Dis 9: 675, 2018

30. Sugiura T, Nagano Y and Noguchi Y: DDX39, upregulated in lung squamous cell cancer, displays RNA helicase activities and promotes cancer cell growth. Cancer Biol Ther 6: 957-964, 2007.

31. Lee S, Baek M, Yang H, Bang YJ, Kim WH, Ha JH, Kim DK and Jeoung DI: Identification of genes differentially expressed between gastric cancers and normal gastric mucosa with cDNA microarrays. Cancer Lett 184: 197-206, 2002.

32. Hellman K, Alaiya AA, Becker S, Lomnytska M, Schedvins K, Steinberg W, Hellström AC, Andersson S, Hellman U and Auer G: Differential tissue-specific protein markers of vaginal carcinoma. Br J Cancer 100: 1303-1314, 2009.

33. Cho B, Lee H, Jeong S, Bang YJ, Lee HJ, Hwang KS, Kim HY, Lee YS, Kang GH and Jeoung DI: Promoter hypomethylation of a novel cancer/testis antigen gene CAGE is correlated with its aberrant expression and is seen in premalignant stage of gastric carcinoma. Biochem Biophys Res Commun 307: 52-63, 2003.

34. Park SY, Kim WJ, Byun JH, Lee JJ, Jeoung D, Park ST and Kim Y: Role of DDX53 in taxol-resistance of cervix cancer cells in vitro. Biochem Biophys Res Commun 506: 641-647, 2018.

35. Cao S, Sun R, Wang W, Meng X, Zhang Y, Zhang N and Yang S: RNA helicase DHX9 may be a therapeutic target in lung cancer and inhibited by enoxacin. Am J Transl Res 9: 674-682, 2017.

36. Ito $\mathrm{S}$ and Koso H: RNA helicase DHX15 acts as a tumor suppressor in glioma. In: Cancer Science. Wiley, Hoboken, NJ, USA. Volume 109, pp 78, 2018.

37. Abdelhaleem M: The novel helicase homologue DDX32 is down-regulated in acute lymphoblastic leukemia. Leuk Res 26 : 945-954, 2002

38. Alli Z, Ho M and Abdelhaleem M: Expression of DHX32 in lymphoid tissues. Exp Mol Pathol 79: 219-223, 2005.

39. Landry JR, Mager DL and Wilhelm BT: Complex controls: The role of alternative promoters in mammalian genomes. Trends Genet 19: 640-648, 2003

40. Tai CL, Pan WC, Liaw SH, Yang UC, Hwang LH and Chen DS: Structure-based mutational analysis of the hepatitis $\mathrm{C}$ virus NS3 helicase. J Virol 75: 8289-8297, 2001

41. Caruthers JM and McKay DB: Helicase structure and mechanism. Curr Opin Struct Biol 12: 123-133, 2002.

42. Schneider S, Hotz HR and Schwer B: Characterization of dominant-negative mutants of the DEAH-box splicing factors Prp22 and Prp16. J Biol Chem 277: 15452-15458, 2002.

43. Alli Z, Ackerley C, Chen Y, Al-Saud B and Abdelhaleem M: Nuclear and mitochondrial localization of the putative RNA helicase DHX32. Exp Mol Pathol 81: 245-248, 2006.

44. Di Liegro CM, Schiera G and Di Liegro I: Regulation of mRNA transport, localization and translation in the nervous system of mammals (Review). Int J Mol Med 33: 747-762, 2014.

45. Alli Z, Ho M, Ackerley C and Abdelhaleem M: Characterization of murine Dhx32. Exp Mol Pathol 83: 115-118, 2007.

46. Iborra FJ, Kimura $\mathrm{H}$ and Cook PR: The functional organization of mitochondrial genomes in human cells. BMC Biol 2: 9, 2004.
47. Chen Y, Alli Z, Ackerley C, Al-Saud B and Abdelhaleem M: Altered distribution of heat shock protein 60 (Hsp60) with dysregulated expression of DHX32. Exp Mol Pathol 82: 256-261, 2007.

48. Abdelhaleem M, Sun TH and Ho M: DHX32 expression suggests a role in lymphocyte differentiation. Anticancer Res 25 : 2645-2648, 2005.

49. McNeer NA, Philip J, Geiger H, Ries RE, Lavallée VP, Walsh M, Shah M, Arora K, Emde AK, Robine N, et al: Genetic mechanisms of primary chemotherapy resistance in pediatric acute myeloid leukemia. Leukemia 33: 1934-1943, 2019.

50. Alli Z,Nam EH, Beimnet K and Abdelhaleem M: The activationinduced expression of DHX32 in Jurkat T cells is specific and involves calcium and nuclear factor of activated $\mathrm{T}$ cells. Cell Immunol 237: 141-146, 2005.

51. Alli Z, Chen Y, Abdul Wajid S, Al-Saud B and Abdelhaleem M: A role for DHX32 in regulating T-cell apoptosis. Anticancer Res 27: 373-377, 2007.

52. Huang $C$, Liang $X$, Huang $R$ and Zhang Z: Up-regulation and clinical relevance of novel helicase homologue DHX32 in colorectal cancer. Journal of experimental \& clinical cancer research. CR (East Lansing Mich) 28: 11, 2009.

53. Lin H, Liu W, Fang Z, Liang X, Li J, Bai Y, Lin L, You H, Pei Y, Wang F, et al: Overexpression of DHX32 contributes to the growth and metastasis of colorectal cancer. Sci Rep 5: 9247, 2015.

54. Lin H, Fang Z, Su Y, Li P, Wang J, Liao H, Hu Q, Ye C, Fang Y, Luo Q, et al: DHX32 promotes angiogenesis in colorectal cancer through augmenting $\beta$-catenin signaling to induce expression of VEGFA. EBioMedicine 18: 62-72, 2017.

55. Wang M, Zhang G, Wang Y, Ma R, Zhang L, Lv H, Fang F and Kang X: DHX 32 expression is an indicator of poor breast cancer prognosis. Oncol Lett 13: 942-948, 2017.

56. Pajares MJ, Ezponda T, Catena R, Calvo A, Pio R and Montuenga LM: Alternative splicing: An emerging topic in molecular and clinical oncology. Lancet Oncol 8: 349-357, 2007.

57. Zhan R, Leng X, Liu X, Wang X, Gong J, Yan L, Wang L, Wang Y, Wang X and Qian LJ: Heat shock protein 70 is secreted from endothelial cells by a non-classical pathway involving exosomes. Biochem Biophys Res Commun 387: 229-233, 2009.

58. Lancaster GI and Febbraio MA: Exosome-dependent trafficking of HSP70: A novel secretory pathway for cellular stress proteins. J Biol Chem 280: 23349-23355, 2005.

59. Bol GM, Khan R, Heerma van Voss MR, Tantravedi S, Korz D, Kato Y and Raman V: PLGA nanoparticle formulation of RK-33: An RNA helicase inhibitor against DDX3. Cancer Chemother Pharmacol 76: 821-827, 2015.

60. Heerma van Voss MR, Vesuna F, Bol GM, Afzal J, Tantravedi S, Bergman Y, Kammers K, Lehar M, Malek R, Ballew M, et al: Targeting mitochondrial translation by inhibiting DDX3: A novel radiosensitization strategy for cancer treatment. Oncogene 37: 63-74, 2018

(i) 9 This work is licensed under a Creative Commons Attribution-NonCommercial-NoDerivatives 4.0 International (CC BY-NC-ND 4.0) License. 